\title{
RF Photoinjector Based Time-Resolved MeV Electron Microscopy
}

\section{P. Musumeci}

University of California at Los Angeles Department of Physics and Astronomy, Los Angeles, CA, USA

Transmission electron microscopy (TEM) is one of the primary tools for materials characterization and finds many important applications in various area of scientific research. Over the past two decades, significant advances have been made in the spatial resolution achievable in TEM (now well below $1 \AA \AA$ ) through the implementation of aberration correction [1]. One of the recent trends to further expand the capabilities of TEMs is to dramatically improve the temporal resolution to ultrafast time scales in order to study the dynamics of microscopic processes in real time [2-4].

So far, two approaches for providing temporal resolution to TEMs have been explored. One is the stroboscopic ultrafast electron microscopy (UEM) developed at Caltech [5]. The number of electrons per pulse is reduced to one on average to minimize the collective Coulomb interactions between electrons. This approach has achieved atomic scale spatial resolution and a few hundred femtosecond temporal resolution, but is limited to study reversible processes since the pump-probe event has to be identically reproduced by millions of times for a single image. The other approach is the single-shot method pioneered by Bostanjoglo and co-workers [6] in which each image is taken by an electron pulse containing millions of particles. The most successful example of this approach, the Dynamic Transmission Electron Microscope (DTEM) developed at LLNL [7] has achieved $10 \mathrm{~nm}$ - 15 ns spatialtemporal resolution. A recent DOE workshop [8] identified one of the critical needs for a major new instrumental development in the area of time-resolved electron microscopy to achieve single-shot realspace imaging with a spatial/temporal resolution of $10 \mathrm{~nm} / 10 \mathrm{ps}$.

In this paper we discuss the development of a single shot picosecond time resolved transmission electron microscope (SPTEM) with $10 \mathrm{ps}$ temporal resolution and $10 \mathrm{~nm}$ spatial resolution based on the use of $\mathrm{MeV}$ beams from an RF photoinjector that would improve the current state-of-the-art in temporal resolution in single shot electron microscopy by three orders of magnitude. A throughout design of the instrument has been outlined in a recent Phys. Rev. Applied paper [9]. A schematic of the proposed system is shown in Fig. 1. The final target of the proposed work (which is currently funded under a Phase I STTR with Radiabeam Technologies) is to enable us to take real-space snapshots of irreversible structural changes with few ps shutter speed and tens of nanometer resolution. The key innovations to enable this goal are: (i) the use of high energy (4 MeV) electrons from a high gradient photocathode radiofrequency $(\mathrm{RF})$ electron gun to employ of the highest possible beam brightness in the TEM, and take advantage of the relativistic suppression of space charge interactions; (ii) an X-band RF cavity to dramatically reduce the energy spread of the beam from the RF gun to below 10-5 thus minimizing the effects of chromatic aberrations; (iii) a compact strong electron optical column based on permanent magnet quadrupoles (PMQs) to avoid the issues of large relativistic electron lenses.

Current state of the art RF photoinjectors use $100 \mathrm{MV} / \mathrm{m}$ peak field gradients to generate very high brightness relativistic electron beams. These sources have played a prominent role in the development of the XFEL and have recently been exploited also in the field of ultrafast electron diffraction [10]. RF photoinjectors have already demonstrated [11] the capability to generate sub-ps beams with $\sim$ pC charge and $<30 \mathrm{~nm}$-rad normalized emittance, satisfying the requirements for single shot imaging in a TEM. By 
lengthening the pulse to $10 \mathrm{ps}$ and further increasing the extraction field on the cathode adopting a novel 1.4 cell gun design, it will be possible to further reduce the emittance to $10 \mathrm{~nm}$, thus reducing the divergence at the sample to $<1 \mathrm{mrad}$ and enabling both incoherent shadow and diffraction contrast imaging mechanisms in the proposed microscope.

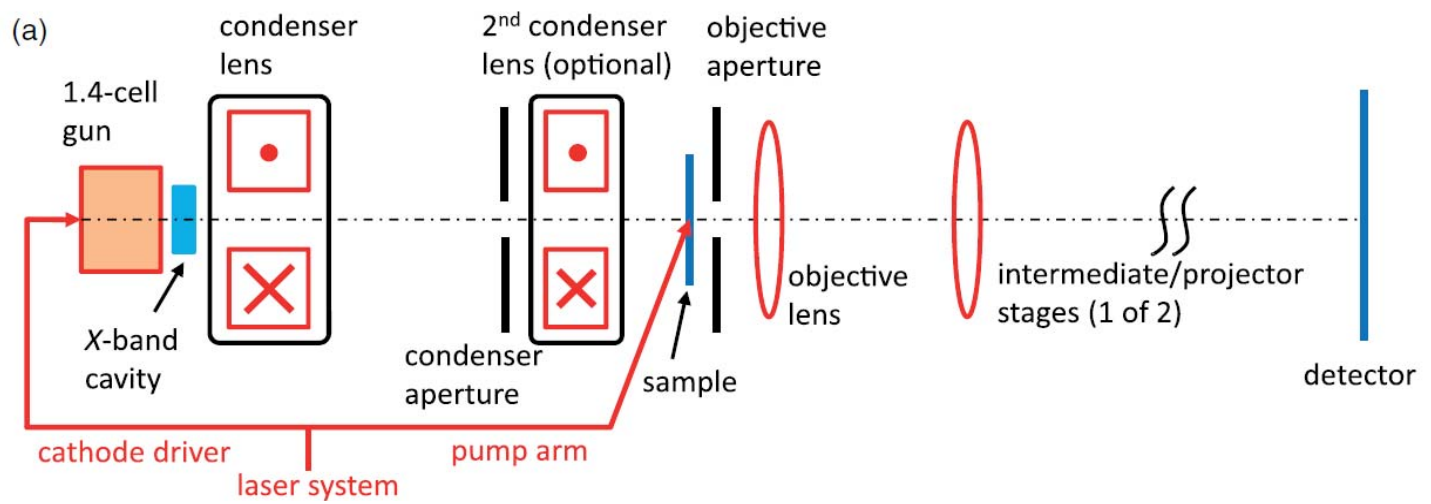

Figure 1: Schematic of the proposed SPTEM system, including an S-band photocathode RF gun, an $X$-band regulation cavity, the main solenoid condenser stage, and the PMQ triplet objective lens.

One particular aspect where further work is needed is certainly the control of the beam energy spread. In order to achieve the required a few tens of nm spatial resolution, the chromatic and spatial aberrations of the focusing column must be minimized and the beam energy spread should be kept below $1 \times 10^{-4}$. This can be achieved introducing an additional RF cavity, operating at X-band, to eliminate the correlated energy spread after the gun.

Another issue is the development of compact electron lenses to replace the large and bulky solenoidal lenses required to focus relativistic beams and thus minimize the propagation distance along the column giving less time to the space charge effects to degrade the image quality. In [9] we devised a solution to provide x12 magnification stage with limited aberrations using a permanent magnet quadrupole triplet. Three magnification stages will be included in the final column for a total magnification of x1000 enabling $10 \mathrm{~nm}$ spatial resolution with standard electron detectors.

\section{References:}

[1] P. E. Batson, N. Dellby, and O. L. Krivanek, Nature 418, 617 (2002).

[2] Future Science Needs And Opportunities For Electron Scattering: Next-Generation Instrumentation And Beyond, DOE workshop March 1-2, 2007.

[3] Dynamic Processes in Biology, Chemistry, and Materials Science: Opportunities for Ultrafast Transmission Electron Microscopy, PNNL workshop June 14-15, 2011.

[4] W. E. King et al., J. Appl. Phys. 97, 111101 (2005).

[5] A. H. Zewail, Science 328, 187 (2010).

[6] O. Bostanjoglo, Advances in Imaging and Electron Physics 121, 1 (2002).

[7] N. D. Browning et al., in Handbook of Nanoscopy, Chapter 9 (2012)

[8] Future of Electron Scattering and Diffraction workshop. http://www.orau.gov/electron2014/

[9] R. K. Li and P. Musumeci. Phys. Rev. Applied, 2, 024003 (2014)

[10] P. Musumeci and R. K. Li, ICFA Beam Dynamics Newsletter \#59 (2012).

[11] R. K. Li et al., Phys. Rev. ST Accel. Beams 15, 090702 (2012). 